\title{
Challenges in measuring measles case fatality ratios in settings without vital registration
}

\author{
K Lisa Cairns ${ }^{1 *}$, Robin Nandy ${ }^{2}$, Rebecca F Grais ${ }^{3}$
}

\begin{abstract}
Measles, a highly infectious vaccine-preventable viral disease, is potentially fatal. Historically, measles case-fatality ratios (CFRs) have been reported to vary from $0.1 \%$ in the developed world to as high as $30 \%$ in emergency settings. Estimates of the global burden of mortality from measles, critical to prioritizing measles vaccination among other health interventions, are highly sensitive to the CFR estimates used in modeling; however, due to the lack of reliable, up-to-date data, considerable debate exists as to what CFR estimates are appropriate to use. To determine current measles CFRs in high-burden settings without vital registration we have conducted six retrospective measles mortality studies in such settings. This paper examines the methodological challenges of this work and our solutions to these challenges, including the integration of lessons from retrospective all-cause mortality studies into CFR studies, approaches to laboratory confirmation of outbreaks, and means of obtaining a representative sample of case-patients. Our experiences are relevant to those conducting retrospective CFR studies for measles or other diseases, and to those interested in all-cause mortality studies.
\end{abstract}

\section{Introduction}

Measles, a highly infectious vaccine-preventable viral disease, is characterized by clustering of cases that occur during cyclical epidemics [1]. In many parts of the world, measles is also a seasonal disease with fewer cases found during the non-measles season [2]. Clinically, the infection is expressed as a maculopapular rash accompanied by fever and at least one of the three "c's": cough, coryza and conjunctivitis; virtually all cases of measles are clinically expressed [3,4]. Measles is a potentially fatal disease [1]. The World Health Organization (WHO) defines a measles-associated death as one occurring within 30 days of rash onset, not obviously due to another cause such as trauma [5].

Historically, measles case fatality ratios (CFRs) have been reported to vary from $0.1 \%$ [1] in the developed world to as high as $30 \%$ among refugee populations $[6,7]$. Current estimates of CFRs used by WHO in endemic countries range between $0.05 \%-6 \%[8-10]$. Factors thought to affect CFR include age [11], intensity of exposure to measles virus (for which household crowding may be seen as a surrogate) [12], measles vaccination status [13], nutritional status [14],

\footnotetext{
* Correspondence: kfc4@cdc.gov

'Global Immunization Division, Centers for Disease Control and Prevention, 1600 Clifton Rd, MS E-05, Atlanta, GA 30333, USA
}

immunodeficiency [15] and access to appropriate case management [16]. Studies conducted in the late $1980 \mathrm{~s}$ demonstrated that supplementation of measles casepatients with vitamin A could decrease measles mortality by as much as $64 \%[17,18]$ leading to recommendations by WHO and United Nations Children's Fund (UNICEF) in 1987 to treat all measles case-patients with vitamin $\mathrm{A}$ in areas where measles CFRs were greater than 1\% [19]. These recommendations, in conjunction with the rollout of Integrated Management of Childhood Illness (IMCI) guidelines in the mid $1990 \mathrm{~s}$ [20] which target pneumonia and diarrhea, might be anticipated to have decreased measles CFRs since the 1990 s. However, few data exist on the extent to which these interventions are used in health facilities, particularly in countries that are highly endemic for measles. Anecdotal evidence from outbreak investigations in Niger, Sudan and South Africa indicate that these interventions are underused [21-24].

Further decrease in CFRs in the past decade may have occurred due to the renewed political will and creation of the Measles Initiative in 2001. WHO and UNICEF developed a comprehensive strategy for sustainable measles mortality reduction with the goal of a $90 \%$ reduction in global measles deaths (compared with 2000 levels) by 2010. 
The four-pronged strategy focuses on improved routine immunization, providing all children with a second dose of measles vaccine delivered either through periodic SIAs or routine services, improved measles case management and careful measles surveillance. This strategy has contributed to reducing the overall burden of measles and has potentially led to decreased CFR linked to earlier detection and improved case management.

Recently the global burden of mortality from measles, critical in prioritizing measles vaccination relative to other health interventions, has been an area of much discussion. A published point estimate from a 2003 analysis of childhood mortality using a proportional mortality model $[25,26]$ differed by hundreds of thousands of deaths from the WHO point estimate for the same period using a static natural history model [27]. A major factor contributing to this discrepancy has been disagreement over which CFRs are appropriate to use in models seeking to estimate global burden of measles deaths, in particular what CFRs are appropriate to use in countries that are highly endemic for measles. In these settings, disease reporting, death surveillance and vital registration tend to be incomplete or non-existent, requiring the collection of primary data to determine disease-specific CFRs.

The recent controversy surrounding measles CFRs in the developing world and the paucity of up-to-date data on this topic have led us to conduct six published studies to determine measles CFR in five countries (Niger [21,24], Sudan [22], Nepal [28], Chad [24] and Nigeria [24], Table 1). Although a recent publication reviewed existing literature to better estimate probable CFRs by geographic region [10], there is little published guidance on the conduct of field studies to determine measles CFR. This paper aims to fill this gap by summarizing both the challenges that are inherent in such studies, and the approach that we advocate given these challenges. Improving the rigor of future studies in this topic area will contribute to more accurate burden of mortality estimates.

\section{Analysis}

We analyzed our experience in conducting measles CFR studies and drew on the published literature to review critical considerations in designing CFR studies. Below, we first review the key components of a CFR study. Second, we summarize and provide recommendations for the conduct of future studies.

\section{Study type}

Prospective disease and death surveillance can be used to determine disease-specific CFRs. However, because highly effective interventions now exist to prevent cases and deaths, it is ethically unacceptable to follow measles outbreaks without offering vaccination to affected communities and optimal case management, including vitamin A supplementation, to case-patients. Further, because this standard of care may not be routinely available, the CFR from such a prospective study may not reflect the true background rate. As a result, the true risk of death may best be studied retrospectively by conducting a cross-sectional survey to determine the number of measles cases that occurred in a defined area during a pre-determined period, and the number of associated deaths.

In conducting a retrospective study of this type, a critical issue is defining the recall period. Guides for the conduct of retrospective mortality surveys and their strengths and weakness have been described in the literature $[29,20]$. The recall period must be long enough such that sufficient cases and deaths occur for a precise CFR calculation, but on the other hand, the longer the recall period, the greater the likelihood of recall bias. Because measles is a disease found primarily in epidemics, identifying an adequate number of measles cases to determine measles CFR with some precision generally requires selecting the recall period to fall within the epidemic. Particularly in settings where measles is highly seasonal, this may restrict the period of interest to only several months. The survey must then be conducted toward the end of or very shortly after the epidemic season. Furthermore, determining measles CFRs retrospectively requires identification of all cases of measles within a household, as well as all deaths occurring in measles case-patients within 30 days of rash-onset. (The definition of a measles case is discussed in the forthcoming section). These requirements presuppose detailed recall, but permit focus on a short period (e.g., 3-6 months) prior to the survey.

\section{Ascertainment of cases and deaths}

To accurately determine CFR, both the number of measles cases and associated deaths occurring during the recall period are needed. Literature on all-cause mortality shows that asking in aggregate about deaths occurring in the past 12-24 months results in 30\%-40\% under-reporting of deaths [30,31]. In addition, differential reporting by both age and gender [32] occurs, with the deaths most frequently omitted variously reported as those among young children [33] and those among children aged 5-15 years [30]. These findings on omission of childhood deaths have led to recommendations in the literature that mothers or other household women serve as respondents [34]. An additional difficulty concerns ascertaining the exact age of household members. Births and deaths are not routinely recorded and age is almost always imprecise. Further, rather than reporting deaths 


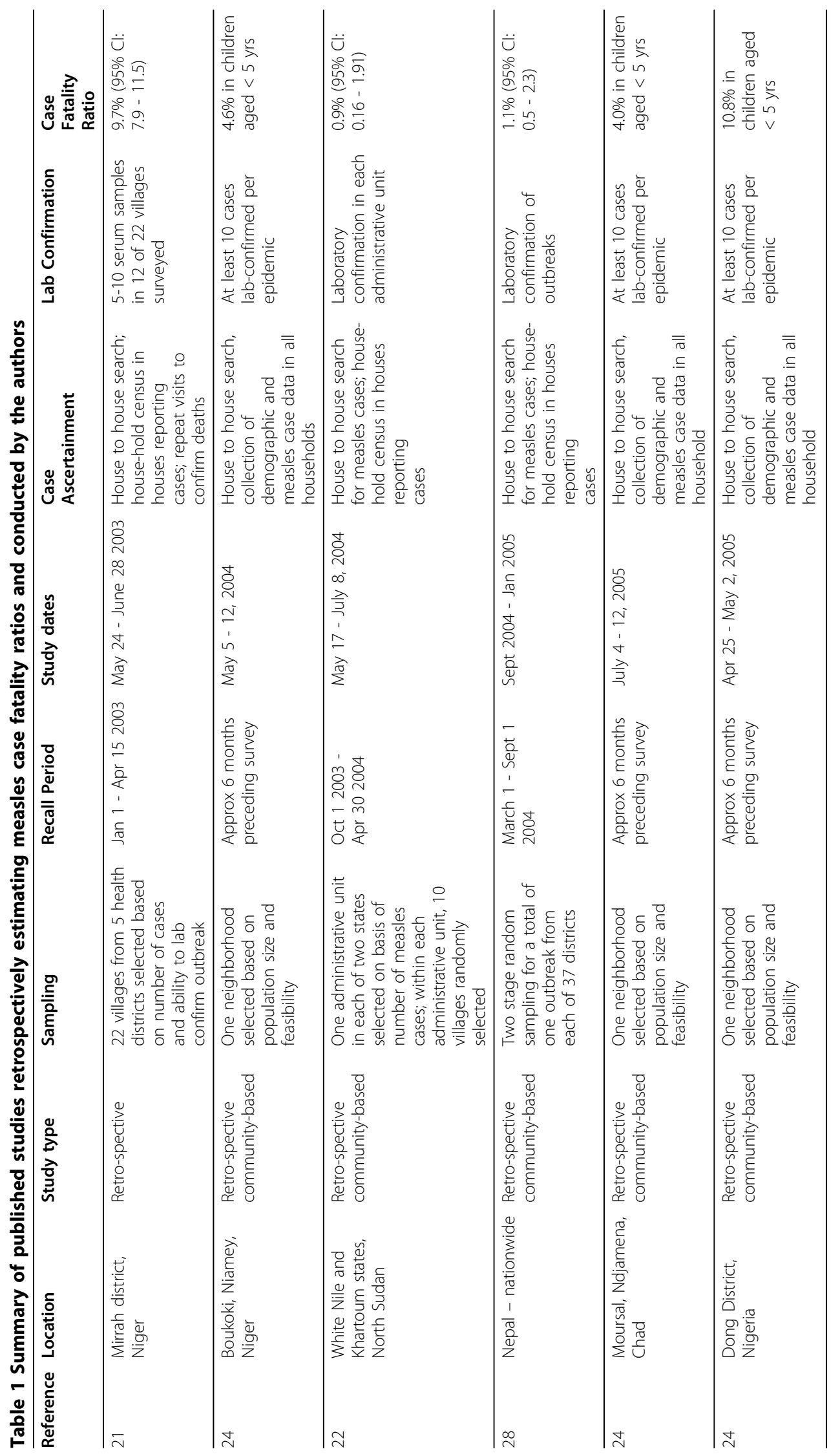


in aggregate, household enumeration should be conducted in order to permit every individual present in the household at the beginning of the recall period, or who joined the household during the recall period, to be accounted for $[35,36]$. We were unable to find literature on the accuracy of retrospective determination of measles cases. However, one might assume that steps recommended to minimize omission of deaths might also minimize the omission of cases.

A further challenge in conducting retrospective studies of measles is ensuring that the illness studied is indeed measles. Misclassification of rash-fever illness will result in inaccurate CFR estimates. Ensuring that the disease is measles is straightforward if cases have been laboratory confirmed. However, when this is not the case, it is necessary to rely upon history.

\section{Laboratory confirmation of measles cases}

Although measles has distinctive clinical features, it can be mistaken for illness due to other causes, most frequently rubella. Thus, measles should be confirmed by collecting serum for testing between days 3 and 28 after rash onset [37]. The implementation of a global measles mortality strategy has led to improved surveillance for measles and the development of a global measles laboratory network $[38,39]$, making laboratory confirmation of measles cases more widely available. However, development of laboratory capacity has been focused in countries that have initiated accelerated measles control, and, even in these settings, laboratories rapidly become overwhelmed during outbreaks [40]. As a result, current WHO recommendations are to limit laboratory confirmation to 5 - 10 specimens per outbreak, although the geographic area implicated (e.g., community, district) is undefined [41]. This recommendation takes into account the fact that the positive predictive value of the clinical case definition for measles increases greatly in the context of an outbreak [42].

The usual WHO laboratory protocol specifies testing for rubella if serum from a suspected measles case tests negative for measles. This has led to the recognition that some outbreaks thought clinically to be due to measles were in fact attributable to rubella, and has also led to confirmation of measles and rubella outbreaks occurring simultaneously in the same communities. In these circumstances, it is difficult to determine by history alone which rash-fever cases were due to measles and which to rubella, thus rendering accurate determination of disease-specific CFRs unfeasible.

In summary, in most measles-endemic settings it is rare that case-patients have laboratory confirmation of measles infection, although it is usually possible to test whether the rash-fever illness circulating is measles. Verifying measles infection in the actual case-patients that we wish to study - i.e., those that have survived 30 days after rash onset and those that have died - remains virtually impossible, as these are patients in whom it is too late to perform confirmatory laboratory testing.

\section{Use of verbal autopsy questions}

As most measles cases in endemic settings are never subjected to laboratory testing, in retrospective studies history must be used to identify cases. Although a vast literature on verbal autopsies exists, these studies have focused primarily on use and validation of verbal autopsy methodology $[43,44]$. Verbal autopsy methodology has been extensively used in retrospective mortality studies addressing measles. Two approaches have been used: a clinical algorithm and the local term for measles. The algorithm using age $\geq 120$ days, fever $\geq 3$ days, and rash to identify measles cases has shown sensitivity ranging from $67 \%-98 \%$, and specificity ranging from $85 \%$ - 99\% [45-47]. WHO recommends the use of either the algorithm or the local term [48]. In studies that compared this algorithm to the use of the local term for measles, the local term was more sensitive and more specific [49].

\section{Obtaining a representative sample of measles case- patients}

In order to obtain a precise estimate of CFR, it is important to include an adequate number of measles cases in the sample. This number will depend upon the precision desired, as well as the pre-study estimate of CFR (Table 2). Estimates of probable CFRs for different regions have been published previously and can be used as a guide for determining sample size [10]. The level of precision desired will depend on the ultimate use of the CFR estimate, but should be as precise as possible. Ideally, the sample should be selected from a sampling frame of all case-patients resident in the geographic area

Table 2 Number of measles case-patients required to retrospectively estimate measles CFR based on expected CFR and desired precision

\begin{tabular}{ccccccc}
\hline \multicolumn{7}{c}{$\mathbf{9 5 \%}$ Confidence Intervals } \\
\hline Expected CFR & $\mathbf{\pm 0 . 5 \%}$ & $\mathbf{\pm 1 \%}$ & $\mathbf{\pm 2} \%$ & $\mathbf{\pm 0} \%$ & $\mathbf{\pm 4} \%$ & $\mathbf{\pm \mathbf { 5 } \%}$ \\
\hline $1 \%$ & 1519 & & & & & \\
\hline $2 \%$ & 3003 & 752 & & & & \\
\hline $3 \%$ & 4452 & 1117 & 279 & & & \\
\hline $4 \%$ & 5866 & 1473 & 369 & 164 & & \\
\hline $5 \%$ & 7246 & 1821 & 456 & 203 & 114 & \\
\hline $10 \%$ & 13641 & 3445 & 864 & 384 & 216 & 138 \\
\hline $15 \%$ & 19215 & 4874 & 1223 & 544 & 306 & 196 \\
\hline $20 \%$ & 23995 & 6109 & 1534 & 682 & 384 & 246 \\
\hline
\end{tabular}

Note: Sample sizes will need to be adjusted based on design effect and response rate 
of interest with rash onset during the period of interest. However, in the absence of a highly sensitive and specific surveillance system, such a sampling frame does not exist. Because measles tends to be highly clustered in both place and time, a sample drawn from the general population may result in a very large number of individuals being surveyed before an adequate number of measles case-patients is identified.

\section{Risk factors}

In addition to estimating CFR, one would like to assess risk factors for increased or decreased CFR among the population studied.

\section{Nutritional status}

Poor nutritional status has been thought to be associated with increased measles CFR, and so one would like to know the nutritional status of measles casepatients at the time of rash onset. However, when conducting a retrospective study in a remote location, this information is rarely available. Instead, some have considered using the nutritional status of family members or of the general community at the time of the survey. However, this approach assumes that nutritional status of those still alive is representative of the nutritional status at disease onset of those who died - which appears unlikely. In some very poor rural communities dependent on subsistence agriculture, there is seasonal fluctuation in the prevalence of wasting. As a result, the community nutritional status during the CFR investigation may not reflect the community nutritional status a few months earlier during the measles outbreak. Logistically, determining current nutritional status adds complexity to field work as enumerators must be trained in anthropometry. If the nutritional status of the community as a whole is to be considered, a population-based sample must be selected for study.

\section{Vaccination status}

Children who received at least one dose of measles-containing vaccine have a lower CFR and reduced complications. Milder measles disease is also associated with a lower CFR in vaccinated children. In some contexts, proof of vaccination can be verified retrospectively from vaccination cards provided through routine vaccination systems or punctual mass vaccination interventions. In this case, the case-patients' vaccination status can be recorded. However, in many instances, card verification is not possible and history must be relied upon. This can be achieved by asking the mother if the child was vaccinated in the upper left arm for measles to avoid confusion with other antigens, but the possibility of response bias is always present. However, previous studies in areas of high measles incidence have shown parental recall to be highly reliable with a predictive value of approximately $95 \%$ [50].

\section{Receipt of vitamin A during measles illness}

Receipt of vitamin A during measles illness has been shown to result in a marked reduction in measles mortality. One would therefore also like to know if measles case-patients received vitamin A while ill and, if so, how many doses were received. Since vitamin A is generally given through health services, it may be possible to determine whether the patient received supplementation through record review. Alternatively, study respondents may be shown a vitamin A capsule and asked whether the case-patient received similar capsules. However, data on vitamin A supplementation during measles illness only support giving two ageappropriate doses of vitamin A separated by 24 hours, as a single dose has not been shown to reduce measles mortality [51]. Record documentation is rarely adequate to determine whether two doses were administered. Furthermore, in countries conducting frequent polio National Immunization Days (NIDs), respondents may confuse the drops of medication from a vitamin A capsule with the drops of oral polio vaccine. In summary, it is quite challenging to obtain accurate information on the receipt of vitamin $\mathrm{A}$ and, if received, the number of doses.

\section{Case management}

Although quality of case management may be an important risk factor for death, adequate evaluation is complex. The major clinical complications of measles leading to death are pneumonia and diarrhea. Evaluation of case management for these includes issues such as appropriate determination of pneumonia and diarrhea, and appropriate choice and dosing of antibiotics (for pneumonia) or appropriate use of oral rehydration solution (for diarrhea).

\section{Our Approach to Estimating Measles CFR}

Taking into account the relevant literature, field experience, and the many challenges mentioned above, we have gradually developed a standardized approach to conducting measles CFR studies.

\section{Study type}

We conducted only retrospective studies for the reasons mentioned previously. In order to best identify all cases and deaths, we used a household census approach, conducting a complete household census of those resident in the household at the beginning of the recall period and using this list to identify those who developed rash illness and died within thirty days of rash-onset. Whenever possible, we limited respondents to mothers or, if they were unavailable, other women within the household. Furthermore, we have limited the recall period to 3-12 months prior to the date of the survey, with the start and end of the period corresponding to a major 
local festival or event. All studies were conducted at the end of the epidemic season or shortly after.

In one study [21] we had the survey supervisors revisit households with reported measles deaths to confirm the numbers of measles cases and deaths. In addition, one of the primary investigators conducted follow-up household visits to ascertain whether the number of measles cases and deaths detected at the first visit matched those at the second visit, prioritizing households in which the questionnaire responses were unclear or incomplete. These follow-up visits comprised approximately $10 \%$ of households.

Despite increasing the likelihood that measles cases and deaths will be accurately detected, this approach has its own difficulties: household enumeration is timeconsuming, and respondents may be suspicious of the motives for enumeration. This is particularly challenging when conducting studies in refugee or displaced populations, in which respondents may infer that benefits are tied to their response.

\section{Ascertainment of cases and deaths}

To maximize the likelihood that the rash-fever illness we are studying is truly measles, we have opted to confirm virologically cases in the same community if the outbreak is still ongoing or, if necessary, in a neighboring community to which the community being studied has epidemiologic links. At times, we have also chosen to restrict the communities that we consider for study to those that have had laboratory confirmation of circulating measles $[21,22,28]$. We have also encountered co-circulating rubella and measles epidemics [28]. In these situations, because of the difficulty in differentiating measles and rubella on clinical grounds, a retrospective study to determine measles CFR may not be feasible.

We initially asked about measles infection using questions extracted from a standardized verbal autopsy questionnaire. However, when we used the algorithm [42-44], we doubted our results because they suggested isolated cases of measles in a poorly vaccinated population, a finding inconsistent with the epidemiology of the disease. We have since chosen to ask about measles infection by using the local term for the disease and then verifying that cases thus identified met the standard clinical case definition for measles, i.e., fever, rash and at least one of the following: cough, coryza and conjunctivitis, in case the term for measles in the local language covers other rash fever illnesses. We recommend this latter approach.

\section{Obtaining a representative sample}

One of the greatest challenges has been identifying a method to select a representative sample of cases.
Ultimately, we have chosen to select randomly communities (or other administrative units) from which several measles cases have been reported, and to conduct comprehensive, active, house-to-house case-searches within these communities. With this approach, all case-patients in communities eligible for study have an equal probability of selection, and findings, including the CFR, can be generalized to the population of case-patients in the communities studied. A confidence interval that accounts for clustering can be calculated. This approach may be criticized for failing to include sporadic cases reported to be measles. As indicated earlier, clustering of cases may lead to increased CFR through increased intensity of viral exposure; focusing solely on clustered cases could theoretically lead to an overestimate of true CFR. However, this is unlikely to have a major influence, because the vast majority of cases occur in outbreak settings and so even if sporadic cases were in fact true measles cases, they contribute relatively little to the total burden of disease. Furthermore, sporadic cases reported to be measles and meeting clinical criteria are far less likely to be true measles cases than clinically or laboratory-confirmed measles cases in laboratory-confirmed outbreak settings, because the positive predictive value of a case definition is much lower when the prevalence of measles is low [52].

\section{Risk factors}

We have generally felt that the logistical difficulties of conducting a community-wide nutritional survey outweighed the potential benefits of doing so. We have chosen to rely instead upon data from the most recent Multiple Indicator Cluster Survey [53] or Demographic and Health Survey [54] to give an indication of probable community nutritional status, while understanding that these data may be neither geographically nor temporally specific to the outbreak under investigation. These surveys are internationally recognized, use standardized methodologies, permit cross-country comparisons, and frequently provide the only information available on country-level nutritional status. If there is no compelling reason to suspect rapid change in nutritional status, estimates from past surveys may give a rough idea of the importance of malnutrition in contributing to elevated CFRs.

We have tried to assess receipt of vitamin A by showing respondents a vitamin A capsule and asking whether the case-patient received similar medication. Although we have also asked about number of doses received, we have questioned respondents' ability to recall such detail, particularly several months after the fact. In one instance, receipt of vitamin A did not show any impact on measles mortality; leading us to question the reliability of the responses we received [21]. 
Table 3 Key recommendations for retrospective measles CFR studies in countries without vital registration

\begin{tabular}{ll}
\hline Survey & Recommendation \\
\hline Timing of survey & Toward end of epidemic or very shortly thereafter \\
\hline Recall period & $3-12$ months with start and end coinciding with major local festival or event \\
\hline Household & Household enumeration should be conducted using past census. Female head of household should be interviewed \\
\hline Laboratory confirmation & Laboratory confirmation of circulating measles in the community under study \\
\hline Case Ascertainment & Clinical algorithm and local term \\
\hline Sampling & Exhaustive house-to house survey in selected communities with reported cases \\
\hline Survey teams & Training on measles, survey methods, case ascertainment with supervisors responsible for no more than 2 to3 teams \\
\hline
\end{tabular}

\section{Case management}

Because of the complexity and additional length of including evaluation of measles case management in our surveys, and because our focus was primarily on determining CFRs rather than evaluating risk factors for death, we chose not to evaluate measles case management in our surveys.

\section{Other considerations}

We ensured strong supervision of survey workers with a supervisor responsible for no more than two or three teams. Conducting complete enumeration of households as well as travelling to randomly-selected but distant locations to conduct studies may add to financial costs. However, we consider these approaches to be critical to the rigor of the studies. Studies using convenience sampling or only considering hospitalized cases may be less costly to conduct, but could lead to biased CFR estimates.

\section{Conclusion}

In conclusion, despite the importance of measuring current measles CFRs in measles-endemic settings for prioritizing measles vaccination relative to other health interventions, the ethical imperative to ensure optimal measles case management limits the generalizability of results from prospective studies. Retrospective studies are in turn limited by recall bias, difficulty ensuring that rash and fever cases are truly attributable to measles, difficulty in assessing important risk factors for increased CFR, and challenges in obtaining a representative sample of measles case-patients. Despite these constraints, we believe that accurate measles CFRs can be obtained from meticulously conducted retrospective studies as we have outlined that take into account the unique characteristics of the disease. Table 3 provides summary guidance for good conduct of retrospective measles CFR studies. Guidelines for conduct of retrospective mortality surveys have been published previously [30,35] and well as guidelines to aid in interpretation [55]. Using these guidelines as a basis, in addition to those presented in Table 3, will help to improve the conduct of retrospective measles CFR studies.

\section{Acknowledgements}

The authors wish to thank the many communities and field teams participating in the conduct of the surveys discussed here.

\section{Author details}

'Global Immunization Division, Centers for Disease Control and Prevention, 1600 Clifton Rd, MS E-05, Atlanta, GA 30333, USA. ${ }^{2}$ Health Section, UNICEF, 3 UN Plaza, New York, NY 10017, USA. Epicentre, 8 rue Saint Sabin, Paris, France 75011.

\section{Authors' contributions}

KLC drafted the manuscript. RN and RFG revised the manuscript critically for important intellectual content. All authors read and approved the final manuscript.

\section{Competing interests}

The authors declare that they have no competing interests.

Received: 15 January 2009 Accepted: 19 July 2010

Published: 19 July 2010

\section{References}

1. Perry RT, Halsey NA: The clinical significance of measles. J Infect Dis 2004, 189(Suppl 1):S4-16.

2. Cliff A, Haggett P: Smallman-Raynor. Measles. An Historical Geography Oxford: Blackwell Publishers 1993, 272-301.

3. Babbot FL, Gordon JE: Modern measles. Am J Med Sci 1954, 228:334-61.

4. Black FL: Measles. Viral infections of humans: Epidemiology and control New York: Plenum publishingEvans AS, 3 1989, 451-65.

5. World Health Organization: Generic protocol for determining measles case fatality rates in a community, either during an epidemic or in a highly endemic area., WHO/EPI/GEN/93.3.

6. Shears P, Berry AM, Murphy R, et al: Epidemiological assessment of the health and nutrition of Ethiopian refugees in emergency camps in Sudan. BMJ 1985, 295:314-18.

7. Porter JDH, Gastellu-Etchegorry M, Navarre I, et al: Measles outbreaks in the Mozambican refugee camps in Malawi: The continued need for an effective vaccine. Int J Epidemiol 1990, 19(4):1072-77.

8. Stein CE, Birmingham M, Kurian $M$, et al: The global burden of measles in the year 2000 - a model that uses country-specific indicators. J Inf Dis 2003, 187(Suppl 1):S8-14.

9. Wolfson LJ, Strebel PM, Gacic-Dobo M, Hoekstra EJ, McFarland JW, Hersh BS, Measles Initiative: Has the 2005 measles mortality reduction goal been achieved? A natural history modeling study. Lancet 2007, 369(9557):191-200.

10. Wolfson L, Grais RF, Luquero FJ, Birmingham ME, Strebel PF: Estimates of Measles Case Fatality Ratios: A Comprehensive Review of CommunityBased Studies. Int J Epidemiol 2009, 38(1):192-205.

11. Barkin RM: Measles mortality: a retrospective look at the vaccine era. Am J Epidemiol 1975, 102:341-49. 
12. Aaby P, Bukh J, Lisse IM, et al: Further community studies on the role of overcrowding and intensive exposure on measles mortality. Reviews of infectious diseases 1988, 10(2):474-77.

13. Clemens JD, Chakraborty J, et al: Measles vaccination and childhood mortality in rural Bangladesh. Am J Epidemiol 1988, 128:1330-39.

14. Nieberg P, Dibley MJ: Risk factors for fatal measles infections. Int J Epidemiol 1986, 15:309-11.

15. Kaplan LJ, Daum RS, Smaron M, et al: Severe measles in immunocompromised patients. JAMA 1992, 267:1237-41.

16. D'Souza RM, D'Souza R: Vitamin A for treating in children (Cochrane Review). The Cochrane Library Oxford: Update Software 2003, 3.

17. Barclay AJG, Foster A, Sommer A: Vitamin A supplements and mortality related to measles: a randomized clinical trial. BMJ 1987, 282:294-6.

18. Fawzi WW, Chalmers TC, Herrera G, et al: Vitamin A supplementation and child mortality - A meta-analysis. JAMA 1993, 269(7):898-903.

19. World Health Organization: EPI Program Report, Geneva 1993.

20. Gove S: Integrated management of childhood illness by outpatient health workers: technical basis and overview. Bull World Health Organ 1997, 75S1:S7-24.

21. Nandy $R$, Handzel $T$, Zaneidou $M$, et al: Case fatality during a measles outbreak in Eastern Niger, 2003. Clin Infect Dis 2006, 42(3):322-8.

22. Coronado F, Musa N, El Tayeb EA, Haithami S, Dabbagh A, Mahoney F, Nandy R, Cairns L: Retrospective measles outbreak investigation - Sudan 2004. J Trop Ped 2006, 52(5):329-334.

23. McMorrow ML, Gebremedhin G, Van den Heever J, Kezaala R, Harris BN, Nandy R, Strebel P, Jack A, Cairns KL: Measles outbreak in South Africa, 2003-2005. S Afr Med J 2009, 99(5):314-9.

24. Grais RF, Dubray C, Gerstl S, Guthmann JP, Djibo A, Nargaye KD, Coker J, Alberti KP, Cochet A, Ihekweazu C, Nathan N, Payne L, Porten K, Sauvageot D, Schimmer B, Fermon F, Burny ME, Hersh BS, Guerin PJ: Unacceptably high mortality related to measles epidemics in Niger Nigeria, and Chad. PLoS Med 2007, 4(1):e16.

25. Black RE, Morris SS, Bryce J: Where and why are 10 million children dying every year? Lancet 2003, 361:2226-34.

26. Morris SS, Black RE, Tomaskovic L: Predicting the distribution of under-five deaths by cause in countries without adequate vital registration systems. Int J Epidemiol 2003, 32:1041-51.

27. Stein $C E$, Birmingham $M$, Kurian $M$, et al: The global burden of measles in the year 2000 - a model that uses country-specific indicators. J Inf Dis 2003, 187(Suppl 1):S8-14

28. Joshi A, Luman E, Nandy R, Subedi B, Liyanage J, Wierzba T: Measles deaths in Nepal: estimating the national case-fatality ratio. Bull World Health Organ 2009, 87:456-465/.

29. Brown V, Checchi F, Depoortere E, Grais RF, Greenough PG, Hardy C, Moren A, Richardson L, Rose AM, Soleman N, Spiegel PB, Sullivan KM, Tatay M, Woodruff BA: Wanted: studies on mortality estimation methods for humanitarian emergencies, suggestions for future research. Emerg Themes Epidemiol 2007, 1;4(1):9.

30. World Food Program: A manual: Measuring and interpreting malnutrition and mortality. WFP: Rome 2005.

31. Tabutin D: Comparison of single and multi-round surveys for measuring mortality in developing countries. Methodologies for the collection and analysis of mortality data. Proceedings of a seminar at Dakar, Senegal; July 7-10, 1981 IUSSP, Liege: Ordina EditionsVallin J, Pollard JH, Heligman L 1984, 12.

32. Blacker JGC: Experiences in the use of special mortality questions in multi-purpose surveys: the single round approach. Data bases for mortality measurement Population Studies No 84, New York, United Nations $1984,80$.

33. Hill AG, David PH: Monitoring changes in child mortality: new methods for use in developing countries. Health Policy Plan 1988, 3(3):214-226.

34. David PH, Bisharat L, Hill AG: Measuring childhood mortality: a guide for simple surveys. UNICEF, Amman, Jordan 1990, ISBN 92-806-0000-2.

35. Measuring Mortality, Nutritional Status and Food Security in Crisis Situations. Smart Methodology Version 12005 [http://smartindicators.org].

36. Rutenberg N, Sullivan J: Direct and indirect estimates of maternal mortality from the sisterhood method. Proceedings of the Demographic and Health Surveys World Conference 1991 Aug 5-7. Washington DC 1969, III.

37. Helfand RF, Heath $J L$, Anderson $L$, et al: Diagnosis of measles with an IgM capture EIA: the optimal timing of specimen collection after rash onset. $J$ Inf Dis 1997, 175:195-9.
38. Featherstone D, Brown D, Sanders R: Development of the Global Measles Network. J Inf Dis 2003, 187(Suppl 1):S264-9.

39. Centers for Disease Control and Prevention: Global measles and rubella laboratory network, January 2004 - June 2005. MMWR 2005, 54(43):1100-4.

40. Yameogo KR, Perry RT, Yameogo A, Kambire C, Konde MK, Nshimirimana D, Kezaala R, Hersh BS, Cairns KL, Strebel P: Migration as a risk factor for measles after a mass vaccination campaign, Burkina Faso, 2002. Int J Epidemiol 2005, 34(3):556-64.

41. World Health Organization: Response to measles outbreaks in measles mortality reduction settings. WHO Geneva, WHO/IVB/09.03.

42. Dietz V, Rota J, et al: The laboratory confirmation of suspected measles cases in setting of low measles transmission: conclusions from the experience in the Americas. Bull World Health Organ 2004, 82(11):852-7.

43. Chandromohan D, Maude GH, Rodrigues LC, Hayes RJ: Verbal autopsies for adult deaths: issues in their development and validation. Int $J$ Epidemiology 1994, 23(2):213-222.

44. Gray HR, Smith G, Barss P: The use of verbal autopsy methods to determine selected causes of death in children. Baltimore: The Johns Hopkins University School of Hygiene and Public Health. Institute for International Programs, Occasional Paper No 101990.

45. Kalter HD, Gray RH, Black R, et al: Validation of post-mortem interviews to ascertain selected causes of death in children. Int J Epidemiol 1990, 19:380-386.

46. Snow R, Armstrong JRM, Forster D, et al: Childhood deaths in Africa: Uses and limitations of verbal autopsies. Lancet 1992, 340:351-355.

47. Mobley CC, Boerma JT, Tituss S, et al: Validation study of verbal autopsy method for causes of childhood mortality in Namibia. J Trop Ped 1996, 42:365-369.

48. World Health Organization: A Standard Verbal Autopsy Method for Investigating Causes of Death in Infants and Children., WHO/CDS/CSR/ ISR/99.4.

49. WHO, Johns Hopkins School of Hygiene and Public Health, The London School of Hygiene and Tropical Medicine: A Standard Verbal Autopsy Method for Investigating Causes of Death in Infants and Children., WHO/ CDS/CSR/ISR/99.4.

50. Samb B, Aaby P, Whittle H, Seck AM, Simondon F: Decline in measles case fatality ratio after the introduction of measles immunization in rural Senegal. Am J Epidemiol 1997, 145(1):51-57.

51. D'Souza RM, D'Souza R: Vitamin A for the treatment of children with measles - A systematic review. J Trop Peds 2002, 48:323-7.

52. Mausner, Kramer : Epidemiology. An Introductory Text W.B. Saunders, 2 1985.

53. Multiple Indicator Cluster Surveys. [http://www.childinfo.org/ mics3_surveys.html], Accessed November 21, 2008.

54. Demographic and Health Surveys. [http://www.measuredhs.com/], Accessed November 21, 2008.

55. Checchi F, Roberts L: Interpreting and Using Mortality Data in Humanitarian Emergencies: A Primer for Non-epidemiologists, Network Paper 52 HPN: London 2005.

doi:10.1186/1742-7622-7-4

Cite this article as: Cairns et al:: Challenges in measuring measles case fatality ratios in settings without vital registration. Emerging Themes in Epidemiology 2010 7:4.

\section{Submit your next manuscript to BioMed Central and take full advantage of:}

- Convenient online submission

- Thorough peer review

- No space constraints or color figure charges

- Immediate publication on acceptance

- Inclusion in PubMed, CAS, Scopus and Google Scholar

- Research which is freely available for redistribution 